\title{
Safety and efficacy of high-dose vitamin B6 for treating antipsychotic-induced hyperprolactinemia in male patients with treatment-resistant schizophrenia
}

Chuanjun Zhuo ${ }^{1}$, Yong $\mathrm{Xu}^{2}$, Haibo $\mathrm{Wang}^{3}$, Chunhua Zhou ${ }^{4}$, Hongjun Tian ${ }^{1}$, Jie Liu ${ }^{5}$, Shuli $\mathrm{Xu}^{5}$, Tao Fang ${ }^{1}$, Jiayue Chen ${ }^{5}$, Cong $\mathrm{Yao}^{5}$, Weilaing Yang ${ }^{5}$, Qianchen $\mathrm{Li}^{4}$, Huan $\mathrm{Mao}^{5}$, Anqu Yang 6 , Bo Li ${ }^{6}$, and Yuhui Chen ${ }^{6}$

${ }^{1}$ Tianjin Fourth Central Hospital

${ }^{2}$ Shanxi Medical University

${ }^{3}$ Peking University Health Science Centre

${ }^{4}$ Hebei Medical University

${ }^{5}$ Tianjin Medical University

${ }^{6}$ Tianjin Kangtai Hospital

March 8, 2021

\begin{abstract}
Background and Purpose: This study aimed to investigate the safety and efficacy of high-dose vitamin B6 (vB6) for antipsychoticinduced hyperprolactinemia (AIHP) treatment in male patients with treatment-resistant schizophrenia (TRS). Experimental Approach: In this randomized double-blinded controlled study, patients were randomized (1:1) into a control group given aripiprazole (ARI; $10 \mathrm{mg} /$ day) or an intervention group given vB6 (300 mg/12 h for 16 weeks). Prolactin level, psychotic symptoms [Positive and Negative Syndrome Scale (PANSS)], cognitive function [MATRICS Consensus Cognitive Battery (MCCB)], liver function, kidney function, growth hormone level, micronutrient levels, blood lipids, and adverse secondary effects (ASEs) [Treatment Emergent Symptom Scale (TESS) and Barnes-Akathisia scale] were monitored. Key Results: After a 16-week treatment period, the vB6 group showed a $68.1 \%$ reduction in serum prolactin levels (from 95.52 $\pm 6.30 \mu \mathrm{g} / \mathrm{L}$ to 30.43 $\pm 18.65 \mu \mathrm{g} / \mathrm{L}$ ) while the ARI group showed only a $37.4 \%$ reduction (from $89.07 \pm 3.59 \mu \mathrm{g} / \mathrm{L}$ to $55.78 \pm 7.39 \mu \mathrm{g} / \mathrm{L}$ ). During weeks 1-4, both treatments reduced prolactin similarly. Subsequently, the ARI effect plateaued, while the vB6 effect remained robust. AIHP reduction efficacy of vB6 was associated with baseline prolactin and triglyceride levels, total vB6 dosage, and education level. Conclusion and Implications: Compared with the ARI group, TRS patients given vB6 showed better attenuation of AIHP, lower ASE scores, and improvements in clinical symptoms and cognitive impairments. These results support further consideration of vB6 as a putative treatment for AIHP.
\end{abstract}

\section{Hosted file}

ZhuoCJ2101211_BJP_Feb21_(1)-zhuo-perfect!!!.pdf available at https://authorea.com/users/ 400235/articles/512569-safety-and-efficacy-of-high-dose-vitamin-b6-for-treatingantipsychotic-induced-hyperprolactinemia-in-male-patients-with-treatment-resistantschizophrenia 


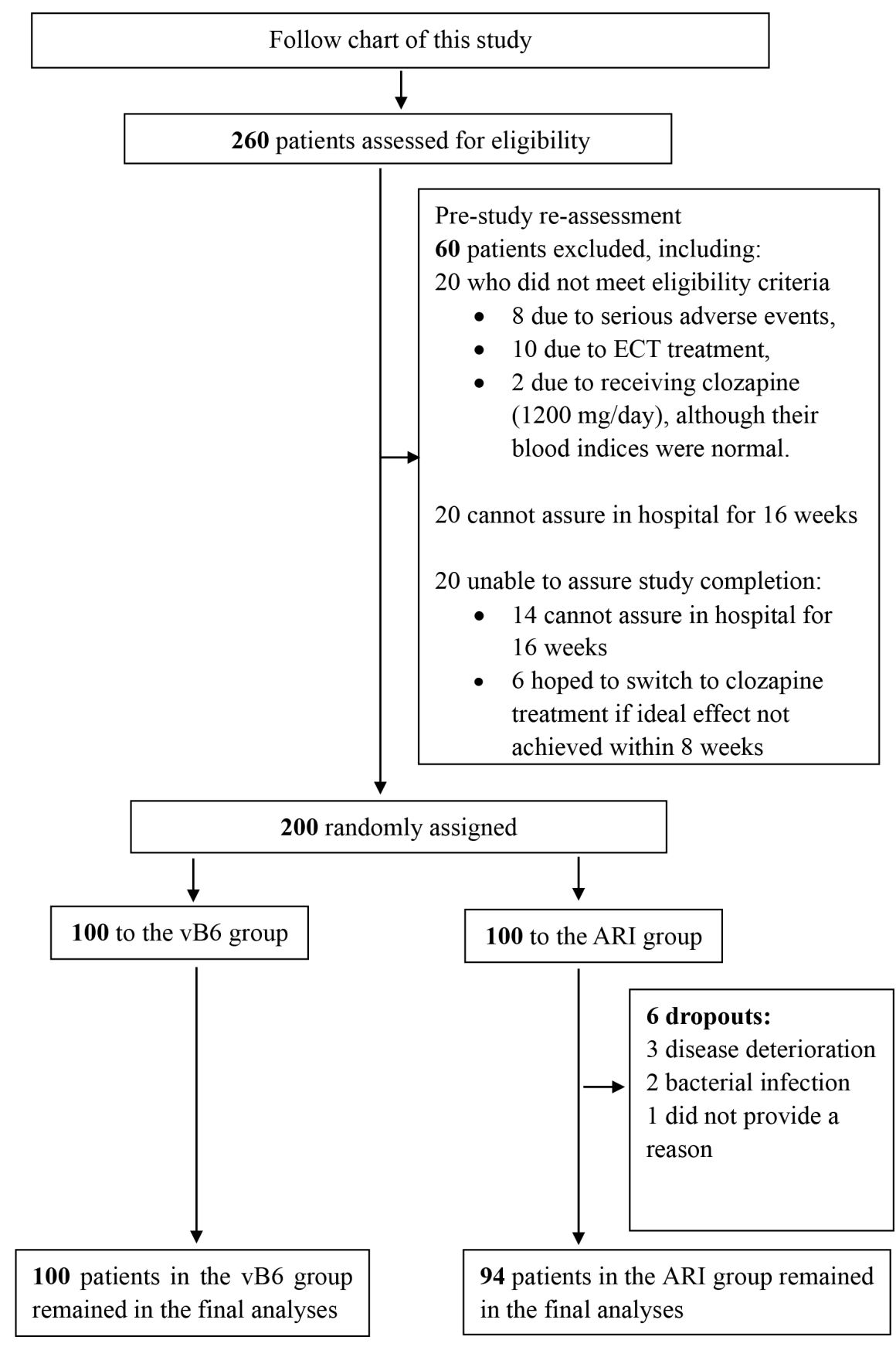




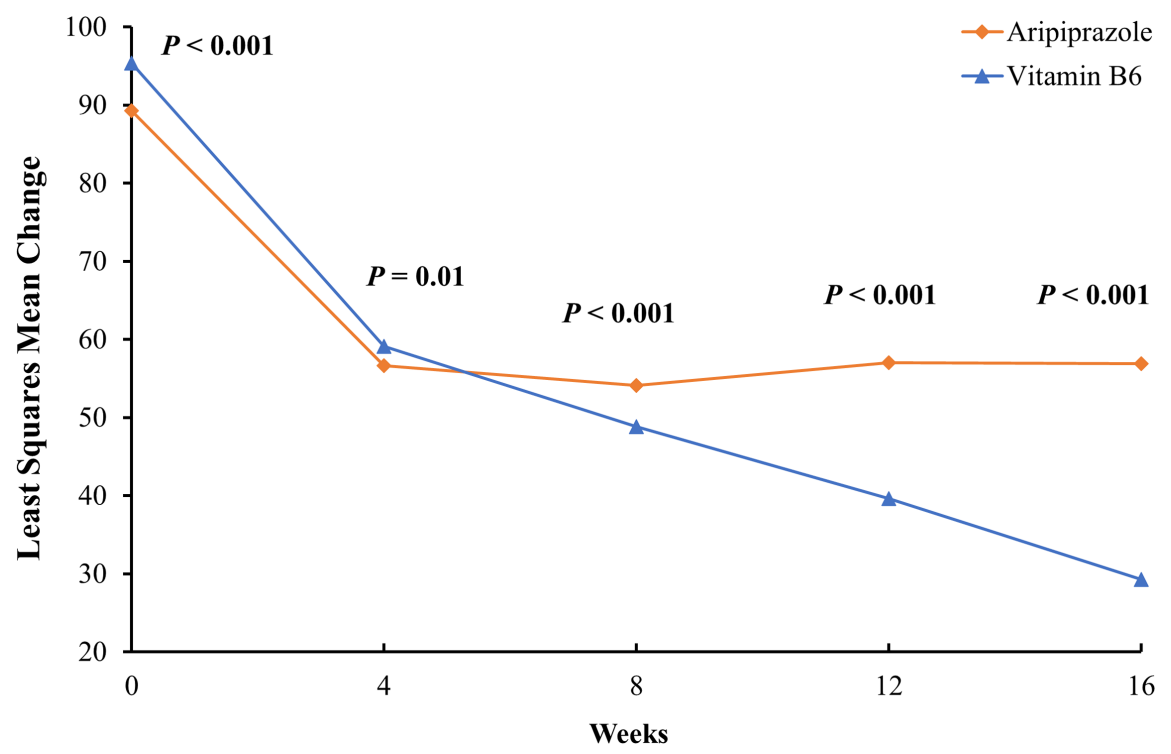

